\section{Regards sur l'économie allemande}

Bulletin économique du CIRAC

$86 \mid 2008$

Varia

\title{
Le pôle biotech de Munich : une « culture de l'innovation »
}

\author{
Horst Domdey et Solène Hazouard
}

Traducteur : Solène Hazouard

\section{OpenEdition \\ Journals}

Édition électronique

URL : http://journals.openedition.org/rea/894

DOI : $10.4000 /$ rea. 894

ISBN : 978-2-8218-0868-3

ISSN : 1965-0787

Éditeur

CIRAC

Édition imprimée

Date de publication : 1 mai 2008

Pagination : 19-24

ISSN : 1156-8992

\section{Référence électronique}

Horst Domdey et Solène Hazouard, « Le pôle biotech de Munich : une «culture de l'innovation » », Regards sur l'économie allemande [En ligne], 86 | mai 2008, document 2, mis en ligne le 01 mai 2010, consulté le 19 avril 2019. URL : http://journals.openedition.org/rea/894 ; DOI : 10.4000/rea.894 


\section{Le pôle biotech de Munich : une "culture de l'innovation "}

\section{Un entretien avec Horst Domdey}

Dans le secteur des biotechnologies, l'Allemagne occupe toujours le premier rang européen : en 2006, le pays comptait près de 500 entreprises dans ce secteur, regroupées le plus souvent au sein de pôles de compétitivité. En première position de ceux-ci figure la BioRegio de Munich construite autour de la localité de Martinsried, quatrième cluster biotechnologique européen après Cambridge, Copenhague/Lund et Zurich/Bâle, et dont le succès repose en grande partie sur le développement de nouvelles molécules. En effet, les deux seuls médicaments issus des biotechnologies allemandes actuellement présents sur le marché ont été conçus par l'entreprise MediGene, implantée sur le site de Martinsried.

A l'heure où on s'interroge en France sur les orientations à donner à la politique des pôles de compétitivité et où un nouveau partage des responsabilités entre l'Etat et les acteurs de la recherche est envisagé, l'approche bottom-up privilégiée outre-Rhin est susceptible d'apporter de nouveaux éclairages sur le rôle de l'initiative privée comme facteur clef de succès dans le développement des clusters.

Nous avons donc demandé au Professeur Horst Domdey, l'un des fondateurs de la société MediGene, président depuis 1998 de la structure de coordination du pôle de Martinsried (Bio ${ }^{\mathrm{M}}$ ), ainsi que du Centre de promotion de l'innovation et de la création d'entreprises (Innovations- und Gründerzentrum Biotechnologie, IZB) d'évoquer avec nous la naissance et l'organisation de la BioRegio de Munich.

Vous êtes l'un des pères du pôle de biotechnologies de Munich. Expliquez-nous comment s'est faite la genèse de ce cluster.

Le cluster n'est pas né ex nihilo, bien qu'il ait vu le jour voici trente ans dans un petit village au sud-ouest de Munich. La proximité de la capitale du Land de Bavière avec ses deux universités et ses centres de recherche, l'attractivité naturelle du site ainsi que la présence de personnel qualifié ont contribué au choix de Martinsried dans l'implantation en 1972 d'un nouvel Institut Max Planck de biochimie, ce qui a, soit dit en passant, brutalement mis fin à la ruralité de Martinsried. Cet Institut Max Planck est né du regroupement de toute une série d'instituts, transférés là pour être mis en réseau. Au fil du temps ont été ensuite intégrés à ce nouvel Institut diverses équipes de l'Université de Munich, dont le Centre d'études génomiques, fondé en 1984. Je suis arrivé pour ma part en 1976 à l'Institut Max Planck de biochimie de Martinsried afin d'y préparer ma thèse de doctorat, avant de poursuivre un temps mes recherches à Lausanne et aux Etats-Unis.

\section{Le cluster s'est donc développé autour de l'Institut Max Planck de biochimie...}

... dans un environnement où les premières entreprises de biotechnologies commençaient à voir le jour, comme Mikrogen. Cette entreprise a été fondée à Martinsried en 1989 par deux chercheurs de l'un des centres de recherche en microbiologie de l'Université Ludwig Maximilian de Munich. Créée par spin-off, elle développe et commercialise des méthodes de diagnostic médical. Elle compte actuellement près de cent collaborateurs et réalise un chiffre d'affaires annuel supérieur à 10 millions $€$. Il est intéressant de relever que cette entreprise a pu être créée sans apport de capital-risque ou de capital extérieur et qu'elle appartient toujours à ses deux fondateurs. Suite à cela sont apparues, de 1992 à 1994, les premières entreprises faisant appel au capital-risque, communément nommées
En 1972, l'Institut Max Planck de biochimie voit le jour à Martinsried

Les premières start ups suivent à partir de 1989 
les « $3 \mathrm{M} »:$ MorphoSys, Micromet et MediGene. J'ai personnellement pris part à la création de MediGene dont le siège a tout naturellement été installé à Martinsried, dans cet environnement porteur en termes de $R \& D$ de biologie moléculaire. La spirale était enclenchée... Voilà comment est né le pôle biotech de Munich.

MediGene AG
La création en 1994 de l'entreprise de biotechnologies MediGene AG s'inscrit dans le prolongement de l'activité de recherche du Centre
d'études génomiques de Munich. Employant près de 175 collaborateurs, la société est aujourd'hui également implantée en Angleterre, à
Oxford et aux Etats-Unis, à San Diego. Cotée à la bourse de Francfort depuis 2000, elle se consacre à la recherche, au développement et
à la commercialisation de nouveaux médicaments pour le traitement de cancers, de maladies de peau et de maladies auto-immunes. Pre-
mière entreprise allemande de biotechnologies, MediGene laissait jusqu'à présent à des entreprises partenaires le soin de distribuer ses
produits. Elle a pour ambition de se lancer en 2008 dans la commercialisation de ses deux médicaments déjà présents sur le marché,
Eligard® et Polyphenon® E-Salbe. Un troisième, Oraceaß, fait actuellement l'objet d'une demande d'autorisation de mise sur le marché.
Malgré un CA de 23,9 millions $€$ en 2007 , l'entreprise enregistre toujours des pertes (-29,9 millions $€)$ liées en grande partie aux coûts
élevés de R\&D, qu'elle espère compenser en 2008 par une forte croissance de CA. (SH)

Entrée tardive des pouvoirs publics dans le jeu des acteurs locaux

Une politique de soutien financier et d'incitation à l'auto-organisation

Mise en place de $\mathrm{Bio}^{\mathrm{M}}$, structure de coordination du pôle
C'est donc la création d'un réseau par des chercheurs, élargi ensuite à des banquiers et des entrepreneurs, qui a mené à la naissance, puis à la croissance progressive du cluster?

C'est exact. Le ministère bavarois de l'Economie n'a décidé que par la suite, en 1995, de mettre en place un Centre de promotion de l'innovation et de la création d'entreprises : I'Innovations- und Gründerzentrum Biotechnologie (IZB). J'ai pris part aux discussions qui ont été menées à l'époque : ce centre pouvait-il apporter de nouvelles perspectives, quelle devait en être la surface ? On a voulu être prudent en fixant dans un premier temps l'étendue des locaux à $800 \mathrm{~m}^{2}$ seulement. Depuis, cet espace a été élargi pour atteindre plus de $15000 \mathrm{~m}^{2}$. Indépendamment de cela, en 1996, les biotechnologies ont connu une impulsion particulière en Allemagne : du fait des moyens financiers limités dont disposait le ministère fédéral de la Recherche, il a été décidé de mettre en place un concours dans le cadre duquel les régions allemandes pouvaient présenter leur candidature, et ce en vue de devenir une "région modèle » dans le domaine des biotechnologies modernes. Nous avons alors pris part à ce concours BioRegio et, à notre plus grande joie, l'agglomération de Munich a fait partie des trois gagnantes parmi les dix-sept régions participantes. Les deux autres lauréats étaient la région de Heidelberg, c'est-à-dire le triangle Rhin-Neckar comprenant les villes de Mannheim, Ludwigshafen et Darmstadt, ainsi que la région de la Rhénanie avec les Universités de Düsseldorf, de Cologne et d'Aix-la-Chapelle.

\section{Qu'attendiez-vous alors des pouvoirs publics ?}

Des félicitations du ministre de la Recherche et une poignée de mains en signe de reconnaissance. Or à notre grande surprise, le gouvernement fédéral nous a annoncé que, dans les régions sélectionnées, la recherche au sein des jeunes entreprises de biotechnologies recevrait un soutien financier de la part du gouvernement fédéral, la priorité dans l'allocation des moyens du ministère fédéral de la Recherche étant accordée aux trois régions gagnantes [en 1996, le financement s'élevait à 25 millions $€$ pour chacun des trois clusters retenus ; SH]. Mais ce qui a été au moins tout aussi important que ce soutien financier, c'est que les régions ont été invitées à s'auto-organiser. II fallait créer les conditions afin de faire émerger les meilleurs concepts. Les BioRegios devaient acquérir la compétence nécessaire pour prendre des décisions de manière autonome. Quant aux start ups en leur sein, elles ont été elles aussi soumises à concurrence, les aides étant attribuées en priorité à celles présentant les concepts les plus porteurs. La répartition de ces moyens financiers limités s'est donc faite sur la base d'une compétition à la fois entre régions et, au niveau de chacune d'entre elles, entre start ups.

\section{Comment avez-vous concrètement réagi à cette invitation à l'auto-organisation ?}

Nous avons alors décidé très rapidement qu'il nous fallait mettre en place un mode d'organisation spécifique pour coordonner nos activités de manière professionnelle. Ainsi, vingt-cinq ans après la fondation de l'Institut Max Planck de biochimie de Martinsried, nous avons créé, en 1997, une plateforme pour mieux fé- 
dérer les acteurs de R\&D et du marché. Nous avons donné à cette société non pas la forme alors habituelle d'une SARL, mais celle d'une SA dans l'espoir d'obtenir des moyens financiers plus conséquents ; ce choix s'est révélé pertinent par la suite. II est vrai que le contexte était porteur : la politique d'innovation du gouvernement fédéral, de même que celle des Länder, comportait alors également un volet visant le développement d'un financement des start ups technologiques par le marché. Cette $\mathrm{SA}$, la $\mathrm{Bio}^{M} A G$, avait pour mission de conseiller, d'informer, d'encourager la mise en place de partenariats, de promouvoir le site économique, d'organiser des manifestations et de mener des activités de lobbying. Mais il s'agissait aussi, et c'était ce qu'il y avait de particulier dans l'organisation du réseau, de proposer un apport de capital d'amorçage aux entreprises. Plus tard, en 2002, nous y avons même intégré un petit fonds de capital-risque.

\section{Ce fonds de capital-risque est-il particulier au cluster munichois ?}

Ce choix que nous avons fait de proposer des financements en termes de capital d'amorçage ou de capital-risque afin de dynamiser la création d'entreprises issues du monde de la recherche nous différencie effectivement d'autres organisations. Nous avons dans notre portefeuille des entreprises dans lesquelles nous avons investi 3,2 millions $€$; nous avons pu lever en outre 200 millions $€$ de capital-risque. Le Land de Bavière y a trouvé également son intérêt car une partie de cet argent lui est revenu sous la forme d'impôts. Nous représentons ainsi une source de revenus aussi bien pour la République fédérale que pour le Land.

\section{Bio $^{M}$ est donc une tête de réseau, l'émanation d'un réseau qui s'est constitué grâce} à l'initiative privée...

Nous avions un domaine d'innovation, un nouveau cluster qui s'est créé de luimême. II n'a pas été mis sur pied de manière artificielle mais il était là, et il a bénéficié ensuite de la mise en place d'une organisation pour le gérer. Cela signifie que les partenaires de ce réseau étaient déjà présents. II ne restait plus qu'à les relier entre eux. Tout l'art de la gestion du réseau consiste à reconnaître très tôt les nouvelles tendances et à y adapter les services proposés. Par exemple, au début, il a beaucoup été discuté des moyens de financement et des possibilités d'entrée en bourse. Plus tard, quand les entreprises ont atteint une certaine taille, s'est posée la question du développement de médicaments, des accords nécessaires en vue de la réalisation de tests et des autorisations de mise sur le marché. Nous avons ainsi construit un réseau multidimensionnel avec des activités multidimensionnelles qui apparaissent puis s'estompent selon les périodes.

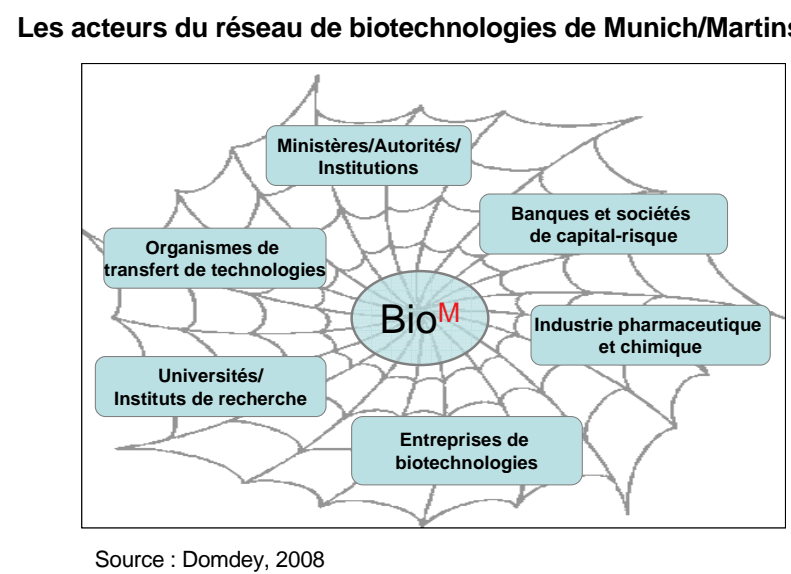

Mais cette Bio ${ }^{\mathrm{M}} \mathrm{AG}$ n'est pas la seule structure en place à Martinsried ?

Cette structure a en effet été scindée en deux à l'automne 2006, lorsqu'un autre domaine d'activité est venu s'ajouter : la gestion du cluster des biotechnologies à l'échelle de l'ensemble de la Bavière. Depuis, les fonctions de gestion du réseau sont exercées par la nouvelle $\mathrm{Bio}^{M} \mathrm{GmbH}$ Biotech Cluster Development, qui est donc non seulement une structure de coordination, mais aussi une sorte de
Création d'un fonds de capital-risque pour les start ups

$\mathrm{Bio}^{\mathrm{M}}$, une structure réticulaire évolutive 
guichet unique pour toute personne œuvrant dans le marché des biotechnologies. La Bio ${ }^{M}$ AG Munich Biotech Development, quant à elle, se concentre exclusivement sur le capital-risque, dont l'amorçage et le coaching. Ces entités, dont les équipes travaillent en étroite collaboration, offrent une complémentarité idéale.

\section{$\mathrm{Bio}^{M}$ : la structure de coordination du réseau de Munich et de la Bavière}

Tandis que l'activité de $\mathrm{Bio}^{\mathrm{M}}$ AG Munich Biotech Development a trait au financement de jeunes entreprises locales innovantes, $\mathrm{Bio}^{\mathrm{M}} \mathrm{GmbH}$ Biotech Cluster Development a pour mission de faciliter le processus de flux d'information et de transfert de technologies au sein du cluster: l'organisation représente un point de contact unique pour les créateurs d'entreprises, les entreprises de biotechnologies ainsi que pour les journalistes et les représentants politiques et industriels. Elle assure la coordination des travaux du réseau munichois avec d'autres pôles de biotechnologies dans le cadre de projets européens, est en charge de l'organisation de séminaires et de congrès scientifiques, ainsi que de la parution régulière d'une newsletter ( $\mathrm{Bio}^{M} \mathrm{Net}$ News »). Enfin, cette organisation dispose d'une fonction de représentation dans le cadre de salons professionnels internationaux et mène par ailleurs des activités de lobbying à l'échelle européenne.

Depuis 2006, la $\mathrm{Bio}^{M} \mathrm{GmbH}$ Biotech Cluster Development, devenue l'instance de représentation de l'industrie des biotechnologies à l'échelle de la Bavière, a pour mission de relier le dispositif de Martinsried aux pôles biotech de Ratisbonne et de Franconie (Bayreuth, Erlangen-Nuremberg, Würzburg). Elle informe notamment les acteurs locaux sur les deux programmes d'innovation et de transfert de technologies, de capital-risque et de coaching proposés par le ministère bavarois de l'Economie, des Infrastructures, des Transports et de la Technologie (StMWIVT) dans le cadre de l'Offensive Clusters du Land (Bourgeois, 2007). II s'agit de BayTOU (Bayerisches Programm zur Förderung technologieorientierter Unternehmensgründungen : soutien aux créateurs d'entreprises et jeunes start ups dans les phases d'établissement d'un business-plan, de développement de nouveaux produits, process et services) et BayTP (Bayerisches Technologieförderungsprogramm : prêts et subventions aux PME désireuses de mettre en œuvre de nouvelles technologies dans leur production et d'innover en termes de produits ou de process). (SH)

Communication active et, surtout, activités de réseau y compris à l'échelle européenne

La mise en réseau des pôles biotech de Bavière

Facteur déterminant le tissu local d'activités économiques ou scientifiques ...

\section{Quels sont les moyens mis en œuvre pour la promotion du site?}

Nous soignons la communication, en organisant des manifestations, ou en tentant d'apparaître au moins une fois par semaine dans la presse locale, ainsi qu'une fois par mois dans les quotidiens suprarégionaux, ou dans les autres médias comme la télévision. Nous organisons également des journées portes ouvertes au sein des entreprises de biotechnologies de Martinsried ou de Munich, nous recevons des groupes de visiteurs et leur présentons le cluster. Mais nous menons aussi un intense lobbying. Nous sommes ainsi à l'origine de la création de la fédération d'entreprises Biotechnologie-Industrie-Organisation Deutschland (BIO Deutschland). II y a trois ans, j'ai lancé un groupe de travail regroupant les pôles régionaux allemands de biotechnologies, nommé Arbeitskreis der BioRegionen Deutschlands ( $A K B R D)$, dont l'objectif était d'aboutir à une coopération entre des régions concurrentes à l'échelle fédérale. Sur la base de ce groupe de travail, nous sommes arrivés à la constitution d'un Conseil des régions européennes de biotechnologies (Council of European Biotech Regions, CEBR), dont fait partie par exemple le groupement d'intérêt public Genopole situé à Evry. Enfin, nous disposons également de groupes de travail plus spécialisés. C'est ainsi que nous essayons d'avoir une certaine influence politique. Mais c'est malheureusement plus difficile que nous ne le pensions.

\section{Comment s'articule le réseau de Martinsried avec les autres réseaux biotech de Bavière?}

Nous avons commencé à intensifier la mise en réseau des sites bavarois de Würzburg, Ratisbonne, Bayreuth...Et nous avons identifié certains problèmes. En premier lieu, malgré le potentiel considérable que représente l'exploitation des biotechnologies blanches (ou biotechnologies industrielles), il reste beaucoup à faire dans ce domaine. Nous avons donc créé un sous-cluster «Biotechnologies blanches » pour lequel nous avons pu rassembler 5 millions $€$ de financement de l'Etat fédéral, 5 millions $€$ à l'échelle du Land et 15 millions $€$ de plus du côté de l'industrie. Par ailleurs, nous souhaitons développer la réalisation d'études cliniques en Bavière. Pour cela, nous avons repéré les centres qui procèdent à ce genre d'études en vue de mettre en place des processus de coopération étroite.

\section{La constitution de la BioRegio de Martinsried a-t-elle été facilitée par la présence} d'universités et d'instituts de recherche autour de Munich ?

Certains facteurs locaux sont indispensables à la constitution d'une culture de l'innovation. Pour notre cluster, cela commence effectivement par les établissements de recherche. A Munich, nous avons la chance d'avoir deux universités de 
pointe qui, avec l'Université de Karlsruhe dans le Land du Bade-Wurtemberg, ont été distinguées "Universités d'Elite » à l'issue du premier tour du concours d'excellence du gouvernement fédéral en octobre 2006. Pourquoi l'Université Ludwig Maximilian et l'Université Technique de Munich sont-elles si performantes? Parce qu'elles sont en situation de concurrence permanente. Par ailleurs, elles sont dotées d'un $\mathrm{CHU}$. Nous disposons en outre de deux Instituts d'enseignement supérieur spécialisés dans les villes de Munich et de Weihenstephan, de trois Instituts Max Planck (de neurobiologie, de biochimie et de psychiatrie) et du Centre Helmholtz de recherche sur l'environnement et la santé. Cela constitue la masse critique nécessaire à l'innovation et explique le taux de $95 \%$ de créations d'entreprises émanant du domaine scientifique. A l'opposé, si vous regardez d'autres sites européens à la pointe dans les biotechnologies, par exemple ceux de Copenhague/Lund ou de Zurich/Bâle, vous verrez que les nouvelles entreprises sont en grande part issues de l'industrie, ce qui est compréhensible : les deux groupes pharmaceutiques Roche et Novartis sont implantés à Bâle.

A l'origine d'un tel cluster, il y a donc toujours un environnement porteur, et une culture favorable au transfert de technologies. Outre la présence d'un tissu scientifique dense et de plateformes de transfert, de bonnes conditions cadres valorisent cette culture de l'innovation. Par exemple pour ce qui est de l'offre en surfaces de bureaux ou de la rapidité avec laquelle la mise en place d'un laboratoire de recherche en génétique peut recevoir l'accord des autorités, et des autorités qui travaillent réellement en collaboration avec les entreprises.

\section{Le cluster de biotechnologies de Munich}

Le quatrième cluster européen s'est nettement développé ces dix dernières années : il comprend actuellement non moins de 191 entreprises dans le domaine des sciences de la vie, qui emploient près de 20000 personnes. Il s'agit d'entreprises pharmaceutiques, de filiales de grands groupes, de CRO (Contract Research Organisations) et de PME de biotechnologies, qui comptent à elles seules pour plus de la moitié des entreprises du réseau et occupent 2500 collaborateurs.

En l'espace de dix ans, la région biotech a connu un essor considérable non seulement grâce au soutien financier du ministère fédéral de la Recherche qui s'élève à 45 millions $€(25$ millions $€$ ont été accordés à l'issue du concours BioRegio, puis près de 20 millions $€$ dans le cadre du programme BioChancePlus), mais aussi et surtout grâce aux 2 milliards $€$ de capitaux privés qui ont permis de financer l'industrie locale des biotechnologies. En résulte une forte croissance du nombre d'entreprises de biotechnologies : tandis qu'en 1997, le cluster comprenait 31 PME pour 370 collaborateurs, ce nombre avait plus que triplé en 2007, où 104 entreprises employaient 2500 personnes. Le chiffre d'affaires de ces entreprises a par ailleurs plus que doublé entre 2001 et 2007, passant de 125 à près de 300 millions €. Un léger bémol cependant en termes de dépenses de R\&D : malgré un pic en 2002 avec un investissement de 225 millions $€$, elles ont connu une légère baisse pour revenir à ce niveau en 2006.

Bien que le volume de financement des entreprises ait fortement augmenté en 2000 (cette augmentation s'explique notamment par deux entrées en bourse qui ont contribué à hauteur de 250 millions $€$ aux investissements, 250 millions $€$ supplémentaires ayant été injectés par des fonds de capital-risque privés cette même année), la région doit faire face à un apport de capitaux insuffisant, le volume de cet apport ayant fortement chuté jusqu'en 2003 pour ne remonter que légèrement depuis. Ainsi, alors que 500 millions $€$ ont été investis dans les biotechnologies en 2000, cette somme ne s'élève plus qu'à 278 millions $€$ en 2006. Le manque de capitaux disponibles sur le marché européen, accompagné d'un trop grand nombre de créations d'entreprises fondées sur des business plans (encore) imprécis et la trop faible incitation pour les scientifiques à commercialiser les résultats de leur recherche constituent les défis que le cluster aura à relever dans les années à venir. (SH)

\section{Quel est le dispositif de transfert dans la région de Munich ?}

Nous disposons des deux premières plateformes de transfert de technologies d'Allemagne, qui sont totalement indépendantes des universités. II s'agit de Max Planck Innovation pour les Instituts Max Planck et d'Ascenion pour les Instituts Helmholtz. S'ajoutent à cela les plateformes de transfert de technologies des universités. Mais celles-ci, qui n'existent que depuis 2002 et qui ont connu des débuts difficiles, en partie en raison d'un sous-financement, sont encore en cours de développement: Par ailleurs, le Centre de promotion de l'innovation et de la création d'entreprises, actuellement présent sur deux sites, contribue activement au transfert de technologies. C'est la seule structure à la fondation de laquelle le Land de Bavière a grandement participé. On pense souvent que le cluster de biotechnologies de Munich a pu se constituer grâce à l'octroi de subventions. Or c'est faux. Les deux Centres de promotion de l'innovation et de la création
Instituts Max Planck et Helmholtz sont les acteurs majeurs du transfert de technologies 
Les compétences managériales des chercheurs sont déterminantes

Bio $^{m}$ : une mission d'aide à l'auto-assistance d'entreprises, qui ont peut-être coûté dans l'ensemble 60 millions $€$, n'ont pas été financés uniquement par des moyens alloués par le Land de Bavière, mais une grande partie de leur financement provient de prêts accordés par des banques privées, actuellement en cours de remboursement.

\section{Dans cette configuration d'acteurs, quel est le rôle joué par les entrepreneurs ?}

Ils représentent un facteur déterminant, car ce sont eux qui font avancer les choses. Les huit entreprises cotées en bourse depuis 1998 avec une capitalisation de 200 à 400 millions $€$ ont fait taire les critiques qui pensaient en 1994, année de la création de MediGene, que ce qui fonctionne aux Etats-Unis, à San Francisco ou à Boston, ne pourrait pas fonctionner en Allemagne, ni en Bavière au sein d'une économie naguère agricole, et encore moins dans un petit village comme Martinsried. La culture de l'innovation est largement présente en Allemagne aussi, mais comme partout, il faut, pour l'activer, des personnalités : des chercheurs et patrons capables de concevoir leur activité comme faisant partie intégrante d'une dynamique ouverte de réseau. Et il faut entre autres des chercheurs animés par l'esprit d'entreprise et dotés de compétences managériales.

Vous avez décrit Bio ${ }^{M}$ comme un réseau de réseaux. Comment voyez-vous son avenir?

Notre mission au sein de $\mathrm{Bio}^{M}$ est de consolider ces multiples maillons d'un même réseau qui sont à la base de la constitution du cluster. II ne faut en effet pas oublier l'existence de sous-réseaux. Par exemple, au sein des entreprises de biotechnologies, les responsables du développement clinique travaillent en réseau avec leurs homologues. II en va de même pour les business developers ou les chefs d'entreprise. II faut donc continuer de multiplier des liens transversaux pour mailler entre eux ces éléments de réseau verticaux et renforcer ainsi, au niveau horizontal, l'interconnexion de ces divers maillons. Ce n'est qu'alors que se constituera un réseau possédant une solidité et une stabilité comparables à celle d'une toile d'araignée. Notre organisation est pour ainsi dire l'araignée qui a généré cette toile, et notre approche est celle de l'aide à l'auto-assistance. Nous espérons que notre travail ne sera un jour plus nécessaire et que nous pourrons, à terme, disparaître. Le succès de $\mathrm{Bio}^{M}$ sera en effet avéré lorsque personne n'aura plus besoin de nos services. C'est-à-dire quand, au sein des réseaux de pôles de biotechnologies allemands et européens, le cluster de Munich sera à même de générer sa propre croissance ; en un mot : de mener la 'vie autonome' de tout pôle d'activités hautement compétitif.

Propos recueillis et traduits par Solène Hazouard

\section{Indications bibliographiques}

DomDEY H., « Das Münchner Biotech Cluster, ein ausgezeichneter Ort der Ideen / Erfahrungen beim Aufbau einer BioRegion », conférence tenue lors de la journée d'étude organisée par le CIRAC et le Deutsch-Französisches Institut (Ludwigsburg), sur le thème $D y$ namiques institutionnelles nouvelles : réseaux territoriaux et soutien aux PME dans le système d'innovation allemand, à Paris le $1^{\text {er }}$ février 2008 (compte rendu de la journée d'étude disponible sur le site www.cirac.u-cergy.fr).

Voir aussi :

BOURGEOIS I., "Bavière: la patiente construction d'une économie performante ", in BOURGEOIS I. (dir.), Allemagne : compétitivité et dynamiques territoriales, Coll. Travaux et Documents du CIRAC, Cergy-Pontoise, juillet 2007

BOURGEOIS I., "Le 'miracle biotechnologique' allemand », Regards sur l'Economie Allemande, $n^{\circ} 57 / 2002$

Guiot C., "L'essor des biotechnologies médicales en RFA », Regards sur l'Economie Allemande, $\mathrm{n}^{\circ} 77 / 2006$

www.bio-m.org ; www.medigene.de ; www.biodeutschland.org ; www.biotechnologie.de ; www.allianzbayerninnovativ.de. 